\title{
ASPECTOS FÍSICOS, PSICOLÓGICOS, COMPORTAMENTAIS E TERAPÊUTICOS DE PACIENTES COM SOBREPESO/OBESIDADE
}

\section{PHYSICAL, PSYCHOLOGICAL, BEHAVIORAL AND THERAPEUTIC ASPECTS OF OVERWEIGHT/OBESITY PATIENTS}

Beatriz Paccini Alves Silva ${ }^{1}$, Isadora Martins Garcia ${ }^{1}$, Ligia Nery Silva Ramos ${ }^{1}$, Tamires Pereira de Souza ${ }^{1}$, Gersika Bitencourt Santos ${ }^{1}$, Roberta Bessa Veloso Silva ${ }^{1}$

\section{RESUMO}

A obesidade e o sobrepeso é uma pandemia ascendente no Brasil e no mundo, capaz de provocar impactos físicos e psicossociais em todas idades e sexos. Assim, realizar a caracterização sociodemográfica, clínica e farmacoterapêutica de pacientes com obesidade e sobrepeso atendidos na Clínica Psicológica da Universidade José do Rosário Vellano tornou-se objetivo deste estudo. Através de um corte transversal, descritivo e exploratório, em uma amostra de 58 pacientes, foi aplicado uma modificação do questionário "Versão Brasileira do Questionário de Qualidade de Vida SF-36" feita pelas autoras. Os resultados indicaram que: (a) os homens apresentaram peso normal com maior frequência do que as mulheres (11,67 pontos percentuais de diferença), além de sobrepeso (4,72 pontos percentuais de diferença), porém a obesidade de grau I e grau II somente foram observadas nas mulheres; (b) com uma diferença de 13,33 pontos percentuais, os homens seguem uma dieta regular mais frequentemente do que as mulheres e, também, disseram se sentirem mais satisfeitos com o peso; (c) 16 participantes $(27,59 \%)$ que relataram terem sofrido algum tipo de violência devido ao peso. $O$ aumento da prevalência de ansiedade e depressão dentre os pacientes obesos é indicativo de que o excesso de peso está atrelado à deterioração da saúde mental.

PALAVRAS-CHAVE: Obesidade. Qualidade de vida. Aspectos psicossociais

\begin{abstract}
Obesity and overweight is a rising pandemic in Brazil and worldwide capable of causing physical and psychosocial impacts in all ages and genders. The objective of this study is performing the sociodemographic, clinical and pharmacotherapeutic characterization of patients with obesity and overweight treated at the Psychological Clinic of Universidade José do Rosário Vellano. Through a crosssectional, descriptive and exploratory, in a sample of 58 patients, a modification of the questionnaire "Versão Brasileira do Questionário de Qualidade de Vida SF-36" made by the authors was applied. The results indicated that: (a) men were more often normal weight than women (11.67 percentage points of difference), in addition to being overweight (4.72 percentage points of difference), but obesity I and II were only observed in women; (b) with a difference of 13.33 percentage points, men follow a regular diet more often than women and also said they felt more satisfied with their weight; (c) 16 participants (27.59\%) reported having suffered some type of violence due to their weight. The increased prevalence of anxiety and depression among obese patients indicates that excess weight is linked to the deterioration of mental health.
\end{abstract}

KEYWORDS: Obesity. Quality of life. Psychosocial aspects

\footnotetext{
1 Universidade José do Rosário Vellano - UNIFENAS

RECIMA21 - Ciências Exatas e da Terra, Sociais, da Saúde, Humanas e Engenharia/Tecnologia
} 


\section{RECIMA21 - REVISTA CIENTÍFICA MULTIDISCIPLINAR ISSN 2675-6218}

ASPECTOS FíSICOS, PSICOLÓGICOS, COMPORTAMENTAIS E TERAPÊUTICOS DE PACIENTES COM SOBREPESO/OBESIDADE Beatriz Paccini Alves Silva, Isadora Martins Garcia, Ligia Nery Silva Ramos, Tamires Pereira de Souza, Gersika Bitencourt Santos, Roberta Bessa Veloso Silva

\section{INTRODUÇÃO}

A partir de uma pesquisa histórica dos padrões alimentares no Brasil, permitiu-se visualizar uma transição da prevalência da desnutrição severa para um cenário em que predominam o excesso de peso e obesidade. Essa é uma tendência global, abrangendo todas as idades, ambos os sexos e representando importante problema de saúde pública (BRASIL, 2006).

A obesidade é definida como o acúmulo excessivo de gordura corporal que acarreta danos à saúde dos indivíduos e provoca prejuízos não apenas físicos, mas também psíquicos. Apresenta-se como um fator de risco para o desenvolvimento de doenças não transmissíveis, e sua prevalência assume trajetória ascendente na população brasileira (BRASIL, 2006).

Em relação aos aspectos psíquicos e cognitivos, a obesidade pode possibilitar doenças neuropsiquiátricas e distúrbios de sono. (SOCIEDADE BRASILEIRA DE PEDIATRIA, 2017).

A obesidade pode influenciar fortemente a autoimagem com percepção de autodesvalorização, afastamento social, exclusão e estigma social, principalmente quando a obesidade é incomum em suas redes sociais, promovendo o aparecimento de uma sintomatologia depressiva, particularmente em um contexto de altas expectativas sociais e padrões de beleza. Por outro lado, os sintomas depressivos podem ajudar a promover o sobrepeso e a obesidade devido a um estilo de vida pouco saudável, como estilo de vida sedentário excessivo, consumo excessivo de álcool e hábitos alimentares pouco saudáveis (MILANO et al., 2020).

Além dos impactos psicossociais, o excesso de peso desencadeia uma série de alterações no organismo humano que dificultam as atividades diárias do indivíduo. A dislipidemia, por exemplo, é uma das complicações proporcionadas pela obesidade, caracterizada pelo aumento de colesterol, triglicerídeos e a redução da fração $\mathrm{HDL}$ colesterol. Isso tudo corrobora para o surgimento de doenças cardiovasculares, endócrino-metabólicas, além de outras comorbidades (WANDERLEY; FERREIRA, 2010).

A obesidade tem caráter complexo e multifatorial, sendo assim, indivíduos obesos necessitam de acompanhamento multiprofissional, os quais devem trabalhar a construção de hábitos alimentares mais saudáveis e estilos de vida mais ativos, a fim de proporcioná-los uma vida mais sadia (DUTRA, 2015). 0 tratamento de pacientes obesos e com sobrepeso é baseado em mudanças comportamentais, dieta e exercícios, com ou sem farmacoterapia ou cirurgia bariátrica, com o objetivo de perder peso e diminuir os fatores de risco (LUCCHETTA et al., 2017).

Somente a partir da identificação dos principais hábitos responsáveis pelo crescente aumento da obesidade no cenário atual, poderemos propor medidas de combate a esta pandemia (PINHEIRO, 2004).

Este estudo tem como objetivo realizar a caracterização sociodemográfica, clínica e farmacoterapêutica de pacientes com obesidade e sobrepeso atendidos em uma clínica de psicologia de uma universidade do sul de Minas Gerais. 


\section{RECIMA21 - REVISTA CIENTÍFICA MULTIDISCIPLINAR ISSN 2675-6218}

ASPECTOS FíSICOS, PSICOLÓGICOS, COMPORTAMENTAIS E TERAPÊUTICOS DE PACIENTES COM SOBREPESO/OBESIDADE Beatriz Paccini Alves Silva, Isadora Martins Garcia, Ligia Nery Silva Ramos, Tamires Pereira de Souza, Gersika Bitencourt Santos, Roberta Bessa Veloso Silva

\section{METODOLOGIA}

Foi conduzido um estudo de corte transversal, descritivo e exploratório para identificação dos hábitos de vida e características clínicas (físicas e psicológicas) e farmacoterapêuticas de pacientes com sobrepeso e obesidade. A população-alvo foi constituída por pacientes atendidos na Clínica Psicológica da Universidade José do Rosário Vellano/UNIFENAS, localizada na cidade de Alfenas, no Sul de Minas Gerais. O estudo foi aprovado pelo Comitê de Ética e Pesquisa em seres humanos sob parecer número 2.523.502.

O instrumento de coleta das informações utilizado para a realização das entrevistas consta da "Versão Brasileira do Questionário de Qualidade de Vida SF-36" (ANEXO I). Este questionário visa detectar os achados clínicos e psicológicos nos pacientes avaliados. Além disso, foi realizada a pesagem dos pacientes e medição de suas alturas com a fita métrica, para permitir o cálculo do Índice de Massa Corpórea (IMC) para então, classificá-lo.

Os participantes foram selecionados de forma aleatória, a partir da listagem dos registros dos pacientes na clínica em estudo. Para tanto, foi utilizada a técnica de amostragem aleatória sistemática, que garantiu a representatividade da amostra.

Os dados coletados foram organizados e apresentados através de tabelas obtendo-se as frequências absoluta, percentual e intervalos de confiança para proporção com $95 \%$ de confiança. As correlações foram submetidas ao teste de independência de qui-quadrado, ao nível nominal de $5 \%$ de significância e, nos casos em que as frequências absolutas das tabelas de contingências foram menores ou iguais a que 5, utilizou-se o teste exato de Fisher (BUSSAB; MORETTIN, 2017).

A análise estatística foi realizada no software $R^{\circledR}$ (R CORE TEAM, 2021).

\section{RESULTADOS}

$\mathrm{Na}$ Tabela 1, pode-se observar que dos 58 participantes, 40 (68,97\%) são mulheres e 18 $(31,03 \%)$, homens. As faixas etárias mais comuns foram, menor ou igual a 20 anos e de 21 a 30 anos, sendo $15(25,86 \%)$ e 20 (34,48\%), respectivamente, seguidas de 31 a 40 anos, 9 (15,52\%), de 41 a 50 anos, $9(15,52 \%)$, de 51 a 60 anos, $4(6,90 \%)$ e acima de 60 anos, 1 (1,72\%). Em relação à escolaridade, 19 (32,76\%), têm ensino superior incompleto; 14 (24,14\%), ensino médio completo; 13 $(22,41 \%)$, fundamental incompleto; 6 (10,34\%), ensino superior completo; 4 (6,90\%), fundamental completo e 2 (3,45\%), ensino médio incompleto. 


\section{RECIMA21 - REVISTA CIENTÍFICA MULTIDISCIPLINAR}

\section{ISSN 2675-6218}

ASPECTOS FísICOS, PSICOLÓGICOS, COMPORTAMENTAIS E TERAPÊUTICOS DE PACIENTES COM SOBREPESO/OBESIDADE Beatriz Paccini Alves Silva, Isadora Martins Garcia, Ligia Nery Silva Ramos, Tamires Pereira de Souza, Gersika Bitencourt Santos, Roberta Bessa Veloso Silva

Tabela 1. Caracterização da amostra, segundo o gênero, faixa etária e escolaridade.

\begin{tabular}{cccc}
\hline Variáveis & $\boldsymbol{n}(\%)$ & IC $(\boldsymbol{p} ; 95 \%)$ & Valor-p \\
\hline Gênero & & & \\
Feminino & $40(68,97 \%)$ & 55,$31 ; 80,10$ & $0,0058^{* *}$ \\
Masculino & $18(31,03 \%)$ & 19,$90 ; 44,69$ & $0,0058^{* *}$ \\
\hline Total & $\mathbf{5 8}$ & & \\
\hline Faixa etária & & & \\
$\leq 20$ anos & $15(25,86 \%)$ & 15,$65 ; 39,29$ & $0,0004^{* *}$ \\
21 a 30 anos & $20(34,48 \%)$ & 22,$82 ; 48,20$ & $0,0256^{*}$ \\
31 a 40 anos & $9(15,52 \%)$ & 7,$77 ; 27,92$ & $<0,01^{* *}$ \\
41 a 50 anos & $9(15,52 \%)$ & 7,$77 ; 27,92$ & $<0,01^{* *}$ \\
51 a 60 anos & $4(6,90 \%)$ & 2,$23 ; 17,55$ & $<0,01^{* *}$ \\
$>60$ anos & $1(1,72 \%)$ & 0,$00 ; 10,46$ & $<0,01^{* *}$ \\
\hline Total & $\mathbf{5 8 ( 1 0 0 , 0 0 \% )}$ & & \\
\hline Escolaridade & & & \\
Fundamental completo & $4(6,90 \%)$ & 2,$23 ; 17,55$ & $<0,01^{* *}$ \\
Fundamental incompleto & $13(22,41 \%)$ & 12,$92 ; 35,60$ & $<0,01^{* *}$ \\
Ensino Médio completo & $14(24,14 \%)$ & 14,$27 ; 37,46$ & $0,0001^{* *}$ \\
Ensino Médio incompleto & $2(3,45 \%)$ & 0,$60 ; 12,95$ & $<0,01^{* *}$ \\
Ensino Superior completo & $6(10,34 \%)$ & 4,$28 ; 21,84$ & $<0,01^{* *}$ \\
Ensino Superior incompleto & $19(32,76 \%)$ & 21,$35 ; 46,46$ & $0,0126^{*}$ \\
\hline Total & &
\end{tabular}

Total

$58(100,00 \%)$

*Significativo ao nível nominal de $5 \%$ de significância, $(p<0,05)$.

**Significativo ao nível nominal de $1 \%$ de significância, $(p<0,01)$.

${ }^{n s}$ Não significativo ao nível nominal de $5 \%$ de significância, $(p>0,05)$.

Quanto aos hábitos de vida (tabagismo e/ou etilismo), verificou-se que 42 (72,41\%), não têm nenhum desses hábitos; 9 (15,52\%), relataram etilismo; 5 (8,62\%), tabagismo e 2 (3,45\%), ambos. Dos 58 participantes, 34 (58,62\%), estavam com o IMC normal; 11 (18,96\%), apresentaram sobrepeso; 6 (10,34\%), obesidade grau I; 4 (6,90\%), obesidade grau II; 2 (3,45\%), baixo peso e 1 (1,72\%), obesidade grau III (TABELA 2).

Observa-se, na Tabela 2, que 43 (74,14\%) seguem uma dieta regular, ao passo que $25,86 \%$, não fazem; 26 (44,83\%), disseram que não praticam nenhum exercício físico, 14 (24,14\%), de 6 a 7 vezes por semana; 12 (20,69\%), de 3 a 5 vezes e $6(10,34 \%)$, de 1 a 2 vezes por semana. Em relação à satisfação com o peso, 29 (50,00\%), disseram estarem satisfeitos e 29 (50,00\%), não. Deve-se chamar a atenção para os $16(27,59 \%)$ que relataram terem sofrido algum tipo de violência devido ao peso. 


\section{RECIMA21 - REVISTA CIENTÍFICA MULTIDISCIPLINAR}

\section{ISSN 2675-6218}

ASPECTOS FísICOS, PSICOLÓGICOS, COMPORTAMENTAIS E TERAPÊUTICOS DE PACIENTES COM SOBREPESO/OBESIDADE Beatriz Paccini Alves Silva, Isadora Martins Garcia, Ligia Nery Silva Ramos, Tamires Pereira de Souza, Gersika Bitencourt Santos, Roberta Bessa Veloso Silva

Tabela 2. Variáveis referentes aos hábitos de vida, a prática de atividade física, satisfação com o peso, entre outras.

\begin{tabular}{|c|c|c|c|}
\hline Variáveis & $n(\%)$ & IC (p; 95\%) & Valor-p \\
\hline \multicolumn{4}{|l|}{ Hábitos de vida } \\
\hline Tabagismo & $5(8,62 \%)$ & 3,$22 ; 19,72$ & $<0,01^{* *}$ \\
\hline Etilismo & $9(15,52 \%)$ & 7,$77 ; 27,92$ & $<0,01^{* *}$ \\
\hline Ambos & $2(3,45 \%)$ & 0,$60 ; 12,95$ & $<0,01^{* \star}$ \\
\hline Nenhum & $42(72,41 \%)$ & 58,$89 ; 82,95$ & $0,0010^{* *}$ \\
\hline $\begin{array}{l}\text { Total } \\
\text { IMC }\end{array}$ & \multicolumn{2}{|c|}{ IMC } & \\
\hline Baixo peso & $2(3,45 \%)$ & 0,$60 ; 12,95$ & $<0,01^{* *}$ \\
\hline Normal & $34(58,62 \%)$ & 44,$96 ; 71,14$ & $0,2373 n s$ \\
\hline Sobrepeso & $11(18,96 \%)$ & 10,$28 ; 31,81$ & $<0,01^{\star *}$ \\
\hline Obesidade I & $6(10,34 \%)$ & 4,$28 ; 21,84$ & $<0,01^{\star *}$ \\
\hline Obesidade II & $4(6,90 \%)$ & 2,$23 ; 17,55$ & $<0,01^{* *}$ \\
\hline Obesidade III & $1(1,72 \%)$ & 0,$00 ; 10,46$ & $<0,01^{* *}$ \\
\hline Total & $58(100,00 \%)$ & & \\
\hline \multicolumn{4}{|l|}{ Dieta regular } \\
\hline Sim & $43(74,14 \%)$ & 60,$71 ; 84,35$ & $0,0004^{* *}$ \\
\hline Não & $15(25,86 \%)$ & 15,$65 ; 39,29$ & $0,0004^{* *}$ \\
\hline Total & $58(100,00 \%)$ & & \\
\hline \multicolumn{4}{|l|}{ Prática de exercícios } \\
\hline Nunca & $26(44,83 \%)$ & 31,$96 ; 58,37$ & $0,5115 n s$ \\
\hline 1 a 2 vezes por semana & $6(10,34 \%)$ & 4,$28 ; 21,84$ & $<0,01^{* *}$ \\
\hline 3 a 5 vezes por semana & $12(20,69 \%)$ & 11,$59 ; 37,72$ & $<0,01^{* *}$ \\
\hline 6 a 7 vezes por semana & $14(24,14 \%)$ & 14,$27 ; 37,46$ & $0,0001^{* *}$ \\
\hline Total & $58(100,00 \%)$ & & \\
\hline \multicolumn{4}{|l|}{ Satisfeito com o peso } \\
\hline Sim & $29(50,00 \%)$ & 37,$54 ; 62,46$ & 1,0000 ns \\
\hline Não & $29(50,00 \%)$ & 37,$54 ; 62,46$ & 1,0000 ns \\
\hline Total & $58(100,00 \%)$ & & \\
\hline
\end{tabular}

Sofreu algum tipo de

violência devido ao peso

\begin{tabular}{lccc} 
Sim & $16(27,59 \%)$ & 17,$05 ; 41,11$ & $0,0010^{* *}$ \\
Não & $42(72,41 \%)$ & 58,$89 ; 82,95$ & $0,0010^{\star *}$ \\
\hline Total & $\mathbf{5 8 ( 1 0 0 , 0 0 \% )}$ \\
\hline${ }^{* *}$ Significativo ao nível nominal de 1\% de significância, $(p<0,01)$. \\
ns Não significativo ao nível nominal de 5\% de significância, $(p>0,05)$.
\end{tabular}




\section{RECIMA21 - REVISTA CIENTÍFICA MULTIDISCIPLINAR}

\section{ISSN 2675-6218}

ASPECTOS FísICOS, PSICOLÓGICOS, COMPORTAMENTAIS E TERAPÊUTICOS DE PACIENTES COM SOBREPESO/OBESIDADE Beatriz Paccini Alves Silva, Isadora Martins Garcia, Ligia Nery Silva Ramos, Tamires Pereira de Souza, Gersika Bitencourt Santos, Roberta Bessa Veloso Silva

$\mathrm{Na}$ Tabela 3 estão apresentadas as variáveis: sistema hematológico, sistema nervoso, sistema cardiovascular, sistema endócrino e medicamentos usados pelos participantes. Em relação ao sistema hematológico, verificou-se apenas um indivíduo com anemia na amostra. Dos 52 indivíduos com alguma patologia relacionada ao sistema nervoso, $69,22 \%$ do total apresentaram ansiedade, e depressão, sendo $18(34,61 \%), 18$ (34,61\%), respectivamente; transtorno bipolar, $5(9,61 \%)$ e, em menor frequência, crises convulsivas, neuropatia, bulimia, TDAH, dependência química e fibromialgia.

Dos 9 indivíduos que apresentaram patologias relacionadas ao sistema cardiovascular, em 7 (77,78\%), verificou-se HAS; 1 (11,11), arritmias e 1 (11,11), hipotensão. Do sistema endócrino, 2 (50,00\%), com diabetes mellitus, 1 (25,00\%), hipotireoidismo e 1 (25,00\%), hipertireoidismo.

Em se tratando dos medicamentos usados, destacam-se os antidepressivos, 11 (26,19\%), seguidos dos hormônios, 7 (16,67); anticonvulsivantes, 4 (9,52\%); hipnóticos/sedativos, 4 (9,52\%); antihipertensivos, (9,52\%); antidiabéticos, (9,52\%); estabilizador de humor, 3 (7,14\%); vitaminas, 2 (4,76\%); antipsicótico, 1 (2,38\%); proteína, 1 (2,38\%) e ferro, 1 (2,38\%), (TABELA 3).

Tabela 3. Variáveis referentes ao sistema hematológico, sistema nervoso, sistema cardiovascular, sistema endócrino e medicamentos usados pelos participantes.

\begin{tabular}{|c|c|c|c|}
\hline Variáveis & $n(\%)$ & IC $(p ; 95 \%)$ & Valor-p \\
\hline \multicolumn{4}{|l|}{ Sistema hematológico } \\
\hline Anemia & $1(100,00 \%)$ & 5,$46 ; 100,00$ & $1,0000 \mathrm{~ns}$ \\
\hline \multicolumn{4}{|l|}{ Sistema nervoso } \\
\hline Ansiedade & $18(34,61 \%)$ & 22,$33 ; 49,16$ & $0,0375^{*}$ \\
\hline Depressão & $18(34,61 \%)$ & 22,$33 ; 49,16$ & $0,0375^{*}$ \\
\hline Transtorno bipolar & $5(9,61 \%)$ & 3,$59 ; 21,79$ & $<0,01^{* *}$ \\
\hline Crises convulsivas & $1(1,92 \%)$ & 0,$10 ; 11,58$ & $<0,01^{* *}$ \\
\hline Neuropatia & $1(1,92 \%)$ & 0,$10 ; 11,58$ & $<0,01^{* *}$ \\
\hline Bulimia & $1(1,92 \%)$ & 0,$10 ; 11,58$ & $<0,01^{* *}$ \\
\hline TDAH & $1(1,92 \%)$ & 0,$10 ; 11,58$ & $<0,01^{* *}$ \\
\hline Dependência química & $1(1,92 \%)$ & 0,$10 ; 11,58$ & $<0,01^{* *}$ \\
\hline Fibromialgia & $1(1,92 \%)$ & 0,$10 ; 11,58$ & $<0,01^{* *}$ \\
\hline
\end{tabular}

\begin{tabular}{cccc}
\hline Sistema cardiovascular & & & \\
HAS & $7(77,78 \%)$ & 40,$19 ; 96,05$ & $0,1824 n s$ \\
Arritmias & $1(11,11)$ & 0,$58 ; 49,33$ & $0,0455^{\star}$ \\
Hipotensão & $1(11,11 \%)$ & 0,$58 ; 49,33$ & $0,0455^{\star}$ \\
\hline Total & $\mathbf{9 ( 1 0 0 , 0 0 \% )}$ & &
\end{tabular}

Sistema endócrino

DM

Hipotireoidismo
$2(50,00 \%)$

$1(25,00 \%)$
15,$00 ; 84,99$

0,5000 ns

1,$32 ; 78,06$

$0,5000 \mathrm{~ns}$ 


\section{RECIMA21 - REVISTA CIENTÍFICA MULTIDISCIPLINAR}

\section{ISSN 2675-6218}

ASPECTOS FíSICOS, PSICOLÓGICOS, COMPORTAMENTAIS E TERAPÊUTICOS DE PACIENTES COM SOBREPESO/OBESIDADE Beatriz Paccini Alves Silva, Isadora Martins Garcia, Ligia Nery Silva Ramos, Tamires Pereira de Souza, Gersika Bitencourt Santos, Roberta Bessa Veloso Silva

\begin{tabular}{cccc} 
Hipertireoidismo & $1(25,00 \%)$ & 1,$32 ; 78,06$ & $0,5000 n s$ \\
\hline Total & $\mathbf{4 ( 1 0 0 , 0 0 \% )}$ & & \\
\hline Medicamentos & $4(9,52 \%)$ & 3,$09 ; 23,54$ & $<0,01^{* *}$ \\
Anticonvulsivantes & $11(26,19 \%)$ & 14,$39 ; 42,32$ & $0,0033^{* *}$ \\
Antidepressivos & $4(9,52 \%)$ & 3,$09 ; 23,54$ & $<0,01^{* *}$ \\
Hipnóticos/Sedativos & $3(7,14 \%)$ & 1,$86 ; 20,55$ & $<0,01^{* *}$ \\
Estabilizador de humor & $1(2,38 \%)$ & 0,$12 ; 14,09$ & $<0,01^{* *}$ \\
Antipsicótico & $4(9,52 \%)$ & 3,$09 ; 23,54$ & $<0,01^{* *}$ \\
Anti-hipertensivos & $4(9,52 \%)$ & 3,$09 ; 23,54$ & $<0,01^{* *}$ \\
Antidiabéticos & $7(16,67)$ & 7,$51 ; 31,96$ & $<0,01^{* *}$ \\
Hormônios & $1(2,38 \%)$ & 0,$12 ; 14,09$ & $<0,01^{* *}$ \\
Proteína & $2(4,76 \%)$ & 0,$83 ; 17,42$ & $<0,01^{* *}$ \\
Vitaminas & $1(2,38 \%)$ & 0,$12 ; 14,09$ & $<0,01^{* *}$ \\
Ferro & $\mathbf{4 2 ( 1 0 0 , 0 0 \% )}$ & & \\
\hline Total & &
\end{tabular}

*Significativo ao nível nominal de $5 \%$ de significância, $(p<0,05)$.

"*Significativo ao nível nominal de $1 \%$ de significância, $(p<0,01)$.

${ }^{n s}$ Não significativo ao nível nominal de $5 \%$ de significância, $(p>0,05)$.

Conforme a Tabela 4, apesar das correlações não terem sido estatisticamente significativas, ( $p>0,05)$, os resultados apresentados na Tabela 5 são significantes. Pode-se observar que o tabagismo ocorreu com uma diferença de 1,94 pontos percentuais a mais nas mulheres. Já o etilismo foi mais frequente nos homens, sendo uma diferença de 1,67 pontos percentuais. Em relação a ambos os hábitos, verificou-se que as mulheres se sobressaíram se comparado aos homens. Quanto a não ter nenhum, com uma ligeira diferença de $2,22 \%$, os homens relataram terem menos destes hábitos do que as mulheres.

Tabela 4. Correlações entre as variáveis de interesse.

\begin{tabular}{c|c}
\hline Variáveis & Valor-p \\
\hline Gênero vs hábitos de vida & $1,0000 \mathrm{~ns}$ \\
Gênero vs IMC & $0,1594 \mathrm{~ns}$ \\
Gênero vs dieta regular & $0,3273 \mathrm{~ns}$ \\
Gênero vs satisfação com o peso & $0,1559 \mathrm{~ns}$ \\
Gênero vs prática de exercícios & $0,6638 \mathrm{~ns}$ \\
físicos & $0,1326 \mathrm{~ns}$ \\
IMC vs escolaridade & $0,3495 \mathrm{~ns}$ \\
IMC vs hábitos de vida & $0,6395 \mathrm{~ns}$ \\
IMC vs prática de exercícios físicos & \\
\hline$n s$ Não significativo ao nível nominal de $5 \%$ de significância, $(\mathrm{p}>0,05)$.
\end{tabular}




\section{RECIMA21 - REVISTA CIENTÍFICA MULTIDISCIPLINAR ISSN 2675-6218}

ASPECTOS FíSICOS, PSICOLÓGICOS, COMPORTAMENTAIS E TERAPÊUTICOS DE PACIENTES COM SOBREPESO/OBESIDADE Beatriz Paccini Alves Silva, Isadora Martins Garcia, Ligia Nery Silva Ramos, Tamires Pereira de Souza, Gersika Bitencourt Santos, Roberta Bessa Veloso Silva

Ao relacionar o IMC e o gênero, deve-se chamar a atenção, na Tabela 5, que os homens apresentaram peso normal com maior frequência do que as mulheres $(11,67$ pontos percentuais de diferença), além de sobrepeso (4,72 pontos percentuais de diferença), porém a obesidade de grau I e grau II somente foram observadas nas mulheres, sendo $6(15,00 \%)$ e $4(10,00 \%)$, respectivamente. Já a obesidade de grau III foi identificada nos homens.

Com uma diferença de 13,33 pontos percentuais, os homens relataram seguirem uma dieta regular mais frequentemente do que as mulheres e, também, disseram se sentirem mais satisfeitos com o peso (1,6 vezes maior o grau de satisfação). Considerando a prática de exercícios físicos, nota-se que as mulheres relataram uma frequência de, 1 a 2 vezes por semana e nunca, maior do que os homens, 5 $(12,50 \%)$ e 19 (47,50\%), respectivamente. Os homens se destacaram na prática de exercícios físicos de 3 a 5 vezes e de 6 a 7 vezes por semana, $4(22,22 \%)$ e $6(33,33 \%)$, respectivamente, se comparado com $8(20,00 \%)$ e $8(20,00 \%)$, respectivamente, nas mulheres. 


\section{RECIMA21 - REVISTA CIENTÍFICA MULTIDISCIPLINAR}

\section{ISSN 2675-6218}

ASPECTOS FísICOS, PSICOLÓGICOS, COMPORTAMENTAIS E TERAPÊUTICOS DE PACIENTES COM SOBREPESO/OBESIDADE Beatriz Paccini Alves Silva, Isadora Martins Garcia, Ligia Nery Silva Ramos, Tamires Pereira de Souza, Gersika Bitencourt Santos, Roberta Bessa Veloso Silva

Tabela 5. Correlações entre o gênero versus os hábitos de vida, IMC, dieta regular, satisfação com o peso e a prática de exercícios físicos.

\begin{tabular}{|c|c|c|}
\hline Variáveis & \multicolumn{2}{|c|}{ Gênero } \\
\hline Hábitos de vida & Feminino $n(\%)$ & Masculino $n(\%)$ \\
\hline Tabagismo & $3(7,50 \%)$ & $1(5,56 \%)$ \\
\hline Etilismo & $6(15,00 \%)$ & $3(16,67 \%)$ \\
\hline Ambos & $3(7,50 \%)$ & $1(5,56 \%)$ \\
\hline Nenhum & $28(70,00 \%)$ & $13(72,22 \%)$ \\
\hline Total & $40(100,00 \%)$ & $18(100,00 \%)$ \\
\hline IMC & & \\
\hline Baixo peso & $1(2,50 \%)$ & $1(5,56 \%)$ \\
\hline Normal & $22(55,00 \%)$ & $12(66,67 \%)$ \\
\hline Sobrepeso & $7(17,50 \%)$ & $4(22,22 \%)$ \\
\hline Obesidade I & $6(15,00 \%)$ & $0(0,00 \%)$ \\
\hline Obesidade II & $4(10,00 \%)$ & $0(0,00 \%)$ \\
\hline Obesidade III & $0(0,00 \%)$ & $1(5,56 \%)$ \\
\hline Total & $40(100,00 \%)$ & $18(100,00 \%)$ \\
\hline \multicolumn{3}{|l|}{ Dieta regular } \\
\hline Sim & $8(20,00 \%)$ & $6(33,33 \%)$ \\
\hline Não & $32(80,00 \%)$ & $12(66,67 \%)$ \\
\hline Total & $40(100,00 \%)$ & $18(100,00 \%)$ \\
\hline \multicolumn{3}{|l|}{ Satisfação com o peso } \\
\hline Sim & $17(42,50 \%)$ & $12(66,67 \%)$ \\
\hline Não & $23(57,50 \%)$ & $6(33,33 \%)$ \\
\hline Total & $40(100,00 \%)$ & $18(100,00 \%)$ \\
\hline \multicolumn{3}{|l|}{ Prática de exercícios físicos } \\
\hline Nunca & $19(47,50 \%)$ & $7(38,89 \%)$ \\
\hline 1 a 2 vezes por semana & $5(12,50 \%)$ & $1(5,56 \%)$ \\
\hline 3 a 5 vezes por semana & $8(20,00 \%)$ & $4(22,22 \%)$ \\
\hline 6 a 7 vezes por semana & $8(20,00 \%)$ & $6(33,33 \%)$ \\
\hline Total & $40(100,00 \%)$ & $18(100,00 \%)$ \\
\hline
\end{tabular}

\section{DISCUSSÃO}

No Brasil, a incidência e prevalência da obesidade e sobrepeso vem crescendo ao longo dos anos nas diferentes idades, sexos e classes econômicas (DIAS, 2017). Os resultados do presente estudo mostram que o excesso de peso constitui um problema de saúde na região de Alfenas, no sul de Minas Gerais. 


\section{RECIMA21 - REVISTA CIENTÍFICA MULTIDISCIPLINAR}

\section{ISSN 2675-6218}

ASPECTOS FíSICOS, PSICOLÓGICOS, COMPORTAMENTAIS E TERAPÊUTICOS DE PACIENTES COM SOBREPESO/OBESIDADE Beatriz Paccini Alves Silva, Isadora Martins Garcia, Ligia Nery Silva Ramos, Tamires Pereira de Souza, Gersika Bitencourt Santos, Roberta Bessa Veloso Silva

Segundo um estudo sobre a prevalência e fatores associados da obesidade na população brasileira, mais da metade dos participantes têm excesso de peso (56,5\% dos homens e $58,9 \%$ das mulheres, e a obesidade foi de $16,8 \%$ entre os homens e $24,4 \%$ entre as mulheres. Tanto a prevalência de excesso de peso como a de obesidade foram superiores no sexo feminino (FERREIRA, 2019). No presente estudo, conduzido no sul de Minas Gerais, foram encontradas taxas de sobrepeso de 17,50\% e $22,22 \%$ entre mulheres e homens, respectivamente, sendo que obesidade foi de $25 \%$ no gênero feminino e 5,56\% no gênero masculino.

São vários os motivos que podem explicar o aumento da prevalência de obesidade e sobrepeso na população geral atualmente. Um deles é a ingestão de álcool: o uso diário ou semanal de álcool, assim como o consumo pesado em uma única ou em várias ocasiões no mês impõe riscos para o ganho de peso (SOUZA, 2020), além de sedentarismo e consumo desmedido de alimentos hipercalóricos. O sobrepeso foi mais prevalente nos homens, no presente estudo, cujo hábito de etilismo era mais frequente.

Além disso, e não menos importante, o comportamento sedentário e a inatividade física, associados a uma alimentação não balanceada (COSTA; MEDEIROS, 2017). Um estudo transversal conduzido em uma escola do Ceará mostrou, em relação à classificação da atividade física, o predomínio de estudantes com comportamento sedentário/irregularmente ativo de 89,7\%. Neste estudo, foi verificado que $44,83 \%$ dos entrevistados não tinham nenhum hábito de prática de exercícios físicos, e ainda $25,86 \%$ destes não controlavam a ingestão calórica, o que pode também explicar o aumento da prevalência de ganho de peso neste grupo (BARBALHO, 2020). Os resultados dessa investigação mostraram, neste estudo, uma mesma inclinação para o predomínio do sexo feminino com atitude sedentária e despreocupação com hábitos alimentares mais saudáveis.

É interessante analisar que, mesmo em pacientes eutróficos, a predominância de doenças psicossomáticas relacionadas ao corpo está presente. A prevalência de distúrbios alimentares está aumentando nos últimos anos, sendo estes mais comuns entre as mulheres e os jovens. Um dos motivos atribuídos para o aumento desses diagnósticos se dá pela influência da mídia em relação ao "corpo perfeito" (HAY, 2020).

Um estudo anterior conduzido entre os alunos na Universidade Estadual de Campinas mostrou que as mulheres estavam insatisfeitas com sua imagem corporal, embora estivessem na faixa de peso ideal, o que denota a preferência por um corpo esguio para se adequar às normas (ZAMAl, 2018). Em um estudo conduzido com universitárias no interior de São Paulo, verificou-se prevalência de $32,24 \%$ de preocupação com o peso, sendo esta condição mais prevalente nas estudantes com excesso de peso (54,54\%) e eutróficas (25,00\%). Na avaliação da silhueta, 80,26\% mostraram-se insatisfeita com o corpo, sendo que a maioria $(59,21 \%)$ deseja perder peso. (SARRO; DIAS, 2018). Isto pode estar atrelado ao fato de que $50 \%$ dos entrevistados relataram estar insatisfeitos com o peso atual, inclusive indivíduos com IMC eutrófico, ainda assim, 74,14\% relataram seguir uma dieta regular. 


\section{RECIMA21 - REVISTA CIENTÍFICA MULTIDISCIPLINAR} ISSN 2675-6218

ASPECTOS FíSICOS, PSICOLÓGICOS, COMPORTAMENTAIS E TERAPÊUTICOS DE PACIENTES COM SOBREPESO/OBESIDADE Beatriz Paccini Alves Silva, Isadora Martins Garcia, Ligia Nery Silva Ramos, Tamires Pereira de Souza, Gersika Bitencourt Santos, Roberta Bessa Veloso Silva

A obesidade é estimulada pela ansiedade através da ativação do sistema nervoso simpático e do eixo hipotálamo-hipófise-supra-renal. A cronicidade dessa ativação resulta no aumento do cortisol, associado a um aumento de peso e alterações de apetite (JACKSON; KIRSCHBAUM; STEPTOE, 2017). Alguns autores elencam uma ligação bidirecional entre a obesidade e a depressão, associando esta última a um risco aumentado de ganho de peso e obesidade, que por sua vez, estão associados a uma maior vulnerabilidade para transtornos depressivos, ligando as duas patologias a desregulação do sistema de estresse e a inflamação central e sistêmica orgânica (MILANO et al, 2020).

A influência das doenças psicossomáticas, em especial a ansiedade e a depressão, faz com que aumente a prevalência de distúrbios alimentares. Neste estudo, 69,22\% dos entrevistados apresentaram, juntos, transtornos ansiosos e depressivos, sendo $34,61 \%$ em cada. Um estudo transversal catarinense encontrou sintomas ansiosos em 17 pacientes $(36,2 \%)$, de forma semelhante, pacientes com sintomas depressivos foram de 16 (34\%). (DELAI, 2020).

\section{CONSIDERAÇÕES FINAIS}

$O$ presente estudo consegue constatar que 0 aumento de peso consegue influenciar negativamente no surgimento de diversas doenças, de âmbitos físico e mental. $O$ aumento da prevalência de ansiedade e depressão dentre os pacientes obesos é indicativo de que o excesso de peso está atrelado à deterioração da saúde mental. No entanto, também é possível concluir que não é apenas o grupo obeso que sofre com distúrbios mentais relacionados ao próprio corpo, pois estes podem ser vistos inclusive em pessoas com IMC normal.

\section{REFERÊNCIAS}

BARBALHO, E. D.; PINTO, F. J.; SILVA, F. R.; SAMPAIO, R. M.; DANTAS, D. S. Influência do consumo alimentar e da prática de atividade física na prevalência do sobrepeso/obesidade em adolescentes escolares. Cadernos Saúde Coletiva, v. 28, n. 1, p. 12-23, jan./mar. 2020. Disponível em: https://doi.org/10.1590/1414-462x202028010181

BRASIL. Obesidade. Brasília: Ministério da Saúde, 2006. 110 p.

COSTA, I. F.; MEDEIROS, C. C.; COSTA, F. D.; FARIAS, C. R.; SOUZA, D. R.; ADRIANO, W. S.; SIMÕES, M. O.; CARVALHO, D. F. Adolescentes: comportamento e risco cardiovascular. Jornal Vascular Brasileiro, v. 16, n. 3, p. 205-13, set. 2017. Disponível em: https://doi.org/10.1590/1677$\underline{5449.011816}$

DELAI, M.; HOHL, A.; MARQUES, E.; PINCELLI, M. RONSONI, M.; VAN DE SANDE-LEE, S. Prevalência de sintomas de ansiedade e depressão em pacientes com diferentes graus de obesidade. Arquivos Catarinenses de Medicina, v. 49, n. 4, p. 86-97, 2020. Disponível em: http://www.acm.org.br/acm/seer/index.php/arquivos/article/view/877/475

DIAS, P. C. Obesidade e políticas públicas: concepções e estratégias adotadas pelo governo brasileiro. Caderno de Saúde Pública (Online), v. 33, n. 7, 2017.

DUTRA, J. R.; SOUZA, S. M. F.; PEIXOTO, M. C. Influência dos padrões de beleza veiculados pela mídia, como fator decisório na automedicação com moderadores de apetite por mulheres no município de Miracema-RJ. Rio de Janeiro: Universidade Iguaçu Campus, 2015. 


\section{RECIMA21 - REVISTA CIENTÍFICA MULTIDISCIPLINAR}

\section{ISSN 2675-6218}

ASPECTOS FíSICOS, PSICOLÓGICOS, COMPORTAMENTAIS E TERAPÊUTICOS DE PACIENTES COM SOBREPESO/OBESIDADE Beatriz Paccini Alves Silva, Isadora Martins Garcia, Ligia Nery Silva Ramos, Tamires Pereira de Souza, Gersika Bitencourt Santos, Roberta Bessa Veloso Silva

FERREIRA, A. P. S.; SZWARCWALD, C. L.; DAMACENA, G. N. Prevalência e fatores associados da obesidade na população brasileira: estudo com dados aferidos da Pesquisa Nacional de Saúde, 2013. Revista Brasileira de Epidemiologia, v. 22, 2019.

HAY, P. Current approach to eating disorders: a clinical update. Internal medicine jornal, v. 50, n. 1, p. 24-29, 2020.

JACKSON, S. E.; KIRSCHBAUM, C.; STEPTOE, A. Hair cortisol and adiposity in a population-based sample of 2,527 men and women aged 54 to 87 years. Obesity, v. 25, n. 3, p. 539-544, 2017.

LUCCHETA, R.; RIVEROS, B. PONTAROLA, R.; RADOMINSKI, R.; OTUKI, M.; FERNANDEZ-LIMOS, F.; CORRER, C. Systematic review and meta-analysis of the efficacy and safety of amfepramone and mazindol as a monotherapy for the treatment of obese or overweight patients. Clinics, v. 72, n. 5, p. 31724, 2017. Disponível em: https://doi.org/10.6061/clinics/2017(05)10

MILANO, W.; AMBROSIO, P.; CARIZZONE, F.; DE BIASIO, V.; DI MUNZIO, W.; FOIA, M. G.; CAPASSO, A. Depression and Obesity: Analysis of Common Biomarkers. Diseases, v. 8, n. 2, p. 23, 2020. Disponível em: https://doi.org/10.3390/diseases8020023

MINISTÉRIO DA SAÚDE. Secretaria de Vigilância em Saúde. Departamento de Análise em Saúde e Vigilância de Doenças não Transmissíveis. Vigitel Brasil 2018: vigilância de fatores de risco e proteção para doenças crônicas por inquérito telefônico: estimativas sobre frequência e distribuição sociodemográfica de fatores de risco e proteção para doenças crônicas nas capitais dos 26 estados brasileiros e no Distrito Federal em 2018. Brasília: Ministério da Saúde, 2019.

MORETTIN, P. A.; BUSSAB, W. O. Estatística Básica. 9. ed. São Paulo: Saraiva, 2017.

PINHEIRO, A. R.; FREITAS, S. F.; CORSO, A. C. Uma abordagem epidemiológica da obesidade. Revista de Nutrição, v. 17, n. 4, p. 523-33, dez. 2004. Disponível em: https://doi.org/10.1590/s1415$\underline{52732004000400012}$

R DEVELOPMENT CORE TEAM. R: A language and environment for statistical computing. Vienna, Austria: R Foundation for Statistical Computing, 2021. ISBN 3-900051-07-0. Disponível em: http://www.Rproject.org.

SARRO, I. O.; DIAS, J. C.; Insatisfação corporal e preocupação com peso em universitárias do município de Bebedouro/SP. Revista Ciências Nutricionais Online, v. 2, n. 2, p. 31-6, 2018.

SOUZA E SOUZA, L. P.; MIRANDA, A. E.; HERMSDORFF, H. H.; SILVA, C. S.; BARBOSA, D. A.; BRESSAN, J.; PIMENTA, A. M. Binge drinking and overweight in brazilian adults - CUME Project. Revista Brasileira de Enfermagem, v. 73, suppl 1, 2018. Disponível em: https://doi.org/10.1590/00347167-2019-0316

WANDERLEY, E. N.; FERREIRA, V. A. Obesidade: uma perspectiva plural. Ciência \& Saúde Coletiva, v. 15, n. 1, p. 185-94, jan. 2010. Disponível em: https://doi.org/10.1590/s1413-81232010000100024 